\title{
Rediscovering clinical utility of QT dispersion: A long-forgotten parameter
}

\author{
Denny Suwanto ${ }^{1,2}$, Ivana Purnama Dewi ${ }^{1,2,3}$, Budi Baktijasa Dharmadjatii ${ }^{1,2}$ \\ ${ }^{1}$ Faculty of Medicine, Airlangga University, Surabaya, Indonesia \\ ${ }^{2}$ Cardiology and Vascular Medicine Department, Dr. Soetomo General Hospital, Surabaya, Indonesia \\ ${ }^{3}$ Faculty of Medicine, Duta Wacana Christian University, Yogyakarta, Indonesia
}

Received: 2021-02-20

Accepted: 2021-03-27

This work is licensed under

reative Commons Attribution 4.0 International License

\section{J Clin Med Kaz 2021; 18(3):4-7}

Corresponding author:

Denny Suwanto.

E-mail: Suwantodenny88@gmail.com;

ORCID: 0000-0002-0224-6712

\section{Abstract}

Clinical and experimental electrophysiological studies have shown the importance of inhomogeneous myocardial repolarization in ventricular arrhythmias' genesis. Increased dispersion of repolarisation provides a substrate for ventricular arrhythmias by generating functional unidirectional block areas, thereby predisposing to reentry. QT dispersion, defined as the difference between the longest and the shortest QT interval on the surface ECG, is a validated measure of repolarisation dispersion. QT dispersion predicts sudden death and ventricular arrhythmias in patients with chronic heart failure from coronary heart disease and various cardiac diseases.

Key words: QT dispersion, inhomogeneous, arrhythmia

\section{Introduction}

The concept of QT dispersion was first introduced by Day et al. in 1990. It was proposed as the dispersion of various ventricular recovery times. Single ECG lead represents the regional myocardial position. Consequently, QT dispersion portrays myocardial repolarization. Clinically, homogenous repolarization on the myocardium is protective toward arrhythmia initiation. Since it was first proposed three decades ago, QT dispersion has been widely studied to assess its clinical applicability.

QT dispersion represents an indirect measurement of ventricular repolarization heterogeneity, with $\mathrm{T}$ wave interval is the primary determinant of QT interval duration. Ventricular repolarization abnormality may disrupt the dynamicity of $\mathrm{T}$ wave loop formation and projections toward ECG, forming variable QT intervals in different leads [1].

\section{Pathophysiology of QT dispersion}

$\mathrm{T}$ wave offset determines the end of the QT interval and, thus, QT dispersion. Therefore, the T wave loop shape and axis are the major factors leading to true variations in the T loop projections' length. Derangement from normal $\mathrm{T}$ loop and axis may significantly affect a single shape of QT interval. Figure 1 showed various T wave morphology and amplitude resulting from different axis projections. $\mathrm{T}$ wave variation results in different offset and formation of QT interval and dispersion.
As a consequence of both different actual lengths and different measurable duration of QT intervals, QT dispersion, if the heart vector becomes perpendicular to one of the leads' axis, two imaginary $\mathrm{T}$ waves of the same magnitude have different offsets (dashed lines). "This results in the dispersion of the QT intervals "true (vertical dashed lines). In comparison, the two T waves' final component is below the threshold level, with different proportions (e.g., with an automatic threshold method). This refers to the measured dispersion (vertical solid line) of the QT intervals, which is separate from the actual dispersion (Figure 1) [2].

Figure 1 - QT Dispersion resulting from both measurable and real duration of QT intervals (5)

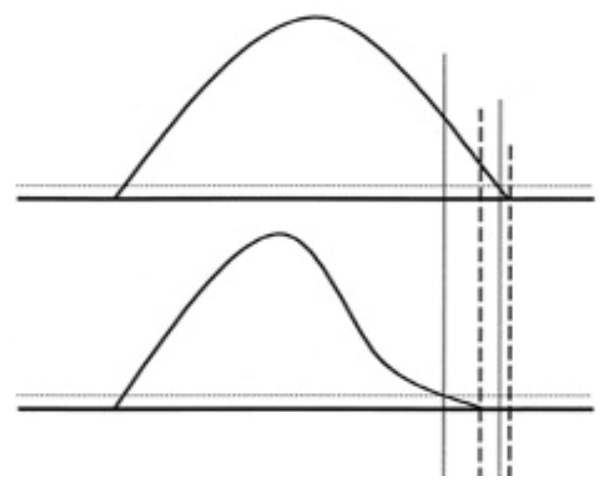




\section{QT dispersion measurement}

Based on standard definition, electrocardiographic QT interval measurement start from the QRS onset to the end of the $\mathrm{T}$ wave, identified as the $\mathrm{T}$ wave returning to the isoelectric line. QT dispersion can be measured from the subtraction of the highest QT interval and lowest QT interval obtained from 12 leads of the ECG simultaneous recording to avoid heart rateinduced QT dynamicity. Determination of T wave offset using the manual method may result in high intra- and interobserver variability. Clinical utilities of automatic repolarization analysis are also not well defined. The intricacy of QT dispersion measurement lies on the $\mathrm{T}$ wave endpoint, especially when the $\mathrm{T}$ wave is abnormal such as biphasic $\mathrm{T}$ wave, $\mathrm{T}-\mathrm{U}$ wave fusion, and $U$ wave presence $[3,4]$.

Several methods are commonly used for $\mathrm{T}$ wave end determination (Figure 2). Threshold methods point to $\mathrm{T}$ wave offset as an intercept of the $\mathrm{T}$ wave with an imaginary line drawn above the isoelectric line. Slope methods determine the T offset as an interception between an imaginary line drawn following the slope of the descending part of the T wave and the isoelectric line. An imaginary line on the T wave slope can be a straight line from the $\mathrm{T}$ wave peak or the steepest tangent line on that slope (Figure 2) [3].

Figure 2 - Methods in Determining T wave Offset (8)
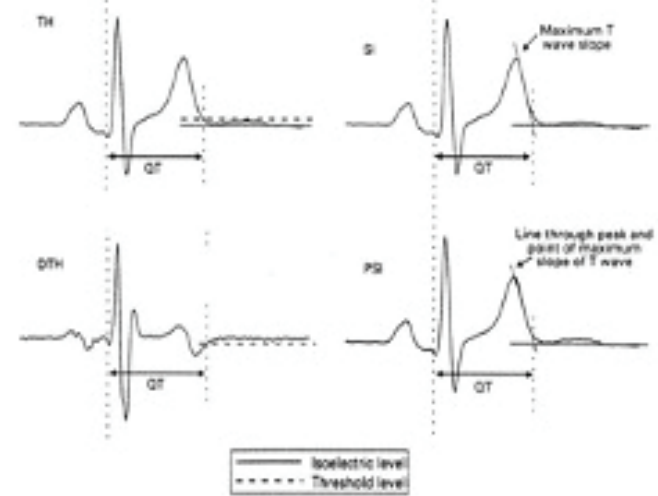

\section{Influence of heart rate on QT dispersion and measurement reliability}

Numerous studies have analyzed the effect of heart rate influence on QT dispersion, known as corrected QT dispersion. Corrected QT dispersion can be calculated using Hodges and Framingham formula used for corrected QT calculation. Several variables, such as heart rate, rhythm, and origin of impulse, can affect QT dispersion. Clinical and experimental data concluded a non-significant correlation between monophasic action potential (MAP) and QT dispersion. It proves QT dispersion as a complex form of electrical impulse influenced by a wide array of factors correction solely toward heart rate may cause a bias [5].

Manual QT dispersion measurements are correlated with $25-40 \%$ of intra and interobserver variability. Even using goldstandard devices such as digitizing boards, the measurement can produce intraobserver variations in $20 \%$ and $10 \%$ of observers. The major source of error in QT dispersion measurement is the determination of the isoelectric line and threshold level [6].

\section{Clinical utilities of QT dispersion}

QT dispersion on a normal individual from literature reviews varies mostly between $40-65 \mathrm{~ms}$ [7]. Rotterdam study with 5,812 healthy adults [age $\geq 55$ years], followed up for 3 to 6.5 years, found its correlation between QT dispersion with cardiac mortality. Strong Heart Study, assessed 1,839 American Indians followed up for $3.7 \pm 0.9$ years, showed the corrected QTc dispersion as a predictor of $34 \%$ of cardiovascular mortality for each $17 \mathrm{~ms}$ increase in QTc dispersion [8].

Several studies have shown a tendency toward increased QT dispersion in various cardiac diseases. Pooled data from 18 studies with a total of 2,525 post-MI patients have shown a tendency toward increased QT dispersion in various cardiac diseases compared to normal subjects [9]. Repolarization abnormalities expressed as QT dispersion might also be founded in ventricular hypertrophy of various origins, including the athlete's heart. However, similar studies have found abnormalities in QT dispersion due to physical training and left ventricular hypertrophy not significantly different from the healthy subjects [10].

Aggressive recommendation toward revascularization procedures in acute Myocardial Infarction (MI) modulates spontaneous dynamicity and regresses infarction size. QT dispersion is increased in acute MI, with mean values ranging from $40 \pm 18$ to $162.3 \pm 64.8 \mathrm{~ms}$. QT dispersion has a trend toward regression on chronic forms of coronary artery disease [11]. There is a significant association of increased QT dispersion on acute MI and ventricular tachycardia incidence (sensitivity $68 \%$, specificity $88 \%$, relative risk $15.7 \quad(\mathrm{p}<0.001)$. The study supported these findings by evaluating the correlation between QT dispersion and infarction size by analyzing viable myocardium by Fluorodeoxyglucose [FDG] uptake and PET scan. Those subjects with increased QT dispersion have a significantly larger infarction size [12].

An indirect relationship of QT dispersion prolongation and infarction size may explain the increased incidence of arrhythmias in acute MI. Increased QT dispersion can be represented as inhomogenous repolarization in the myocardium area. This inhomogeneous as the area can form a substrate for reentrant arrhythmias initiation. Prolongation of the repolarization phase can also induce arrhythmia through the after depolarization mechanism [13].

Several treatments have been correlated with QT dispersion improvement, e.g., reperfusion therapy with the thrombolytic agent, revascularization with the percutaneous coronary intervention, or coronary artery bypass grafting. Restoration of epicardial coronary flow may improve stunned myocardium, reduce scar tissue formation, and induce homogenous repolarization on the myocardial [13-15]. Betablocker treatment in a patient with the long-QT syndrome and candesartan for heart failure and hypertension has also decreased QT dispersion [16].

\section{Prognostic Value of QT Dispersion}

Data from 23 studies involving 1831 patients with various cardiac diseases with and without serious ventricular arrhythmias during their illness showed significantly dispersed QT in patients with arrhythmias, most of them with coronary artery disease. Contrary to Zabel et al., in his study involving 280 consecutive MI survivors followed up for $32 \pm 10$ months, it showed non-associative QT dispersion as a predictor of adverse outcome [15].

Analysis from the ELITE heart failure study comparing losartan and captopril showed a reduction of sudden cardiac death in the losartan arm [17]. The DAMOND-CHF Study's substudies, the UK-HEART study, showed a non-significant association of QT dispersion for outcome predictors in heart failure patients. An analysis of the effect of beta-blockers in 
dilated cardiomyopathy by Oflaz et al. reported a better response in patients with higher QT dispersion ( $137 \pm 52$ vs. $75 \pm 38 \mathrm{~ms})$ [18].

\section{Effect of drugs on QT dispersion and the Risk of Torsades de Pointes [TdP]}

Class IA anti-arrhythmias have the effect of inducing prolongation of ventricular repolarization. In a patient who develops TdP, class IA antiarrhythmics may induce inhomogeneous prolongation of the repolarization phase expressed as increased QT dispersion. Propafenone, disopyramide, and almokalant (blocker of the rapid component of the delayed rectifier, I $\mathrm{Kr}$ ) have been shown to increase QT dispersion, whereas, in one study, intravenous dofetilide did not produce increased QT dispersion [19]. The unique effect of amiodarone [class III antiarrhythmic agent] induced a global QT prolongation by its beta-blockade and potassium channel blocking ability, thereby protecting TdP risk and not being associated with increased QT dispersion [19,20].

Sotalol therapy for six months after acute infarction induces variation in QTc and QTc dispersion. Sotalol is associated with increased maximal QTc and reduced QTc dispersion compared to placebo. This effect is protective toward arrhythmia by inducing homogenous ventricular repolarization. A study by Grimm et al. analyzed QTc dispersion and adjusted QTc dispersion on 52 patients with ventricular arrhythmia receiving amiodarone. It is showed non-significant prolongation of QT dispersion before and after amiodarone administration. ECG and QT dispersion can monitor myocardium response during the drug administration with ventricular repolarization modulation activity. QT dispersion >100 ms during drug usage is an absolute parameter of severe repolarization prolongation and potentially induces arrhythmia by afterdepolarization mechanism [21].

Antiarrhythmic agents are the leading cause of druginduced TdP. As proposed earlier, the QT interval only represents the mean sum of repolarization activities in the ventricular myocardium. Measuring QT dispersion will give additional information on regional repolarization abnormalities that predispose to early afterdepolarization and TdP. Therefore, measurement of QT dispersion is essential during the administration of antiarrhythmics. Class IA agents (quinidine, procainamide, and disopyramide) block both $\mathrm{Na}$ and $\mathrm{K}$ channels, and TdP can occur either at therapeutic or subtherapeutic doses. Quinidine prolongs QT interval by an average of $10-15 \%$ within a week of therapy initiation and carries a $1.5 \%$ risk of inducing TdP. Class III agents are potent IKr blockers and prolong QT interval in a dose-dependent manner [22].

Although no single clinical manifestation can be presented by widely dispersed QT, its presence in the therapy of various antiarrhythmic drugs may signal a predisposition in arising of torsade de Pointes (TdP) or polymorphic ventricular tachycardia.
Various studies have shown that therapy using amiodarone with its unique combination of all four antiarrhythmics classes is associated with the rarity of drug-induced $\mathrm{TdP}$ and superior choice in various arrhythmias [21].

\section{Long QT Syndrome as Model of Congenital Prolong QT Interval}

The long QT syndrome (LQTS) is considered a blueprint for studying repolarization anomalies in humans. Cardiac repolarization abnormalities in long QT syndrome is a pure electrical condition without corresponding structural abnormalities. Prolonged ventricular repolarization's arrhythmogenic ability has been extensively studied in both laboratory models and clinical LQTS patients. It is demonstrated that LQTS mutations can establish a fragile substrate with prolonged action potential duration, where early afterdepolarization and triggered activity can occur in the presence of beta-adrenergic stimulation [23].

These results were then verified in two additional studies, demonstrating that transmural variations in action potential recovery times are crucial in determining the heterogeneity of repolarization in a pharmacologically induced LQTS mode. Torsades-de-Pointes arise when this dispersion is exacerbated by the existence of an altered IKs current (as occurs in the LQT1 subtype) and -adrenergic stimulation. It's worth noting that these latter observations, suggesting the pathogenetic significance of the adrenergic nervous system in LQTS, are consistent with our group's clinical evidence. The baseline inhomogenous repolarization of congenital long QT syndrome is a classic manifestation of QT dispersion.

\section{Conclusion}

The overlapping of values QT dispersion values in normal and pathological cardiac states render any attempt at establishing reference values. Although theoretically, QT dispersion is a parameter in evaluating ventricular repolarization, conflicting study results hinder QT dispersion applicability as a clinical tool. Twelve lead ECGs may provide regional electrical data. However, simple measurement using QT dispersion has a poor ability to extract that information. Further study is needed to explore the difference between the duration of local, global, and interventricular dispersion. It provides information about a dispersed of the distance electrical impulse that is important for arrhythmogenesis.

Disclosures: There is no conflict of interest for all authors.

Acknowledgements: None.

Funding: None.

\section{References}

1. Tokatli A, Yiginer O, Haholu A, Uzun M. Efficiency of quantification of cardiac electrical heterogeneity: Via QT dispersion, transmural dispersion, or both. Biology of Sport. 2015; 32(2):175-6. doi: 10.5604/20831862.1145079.

2. Malik M, Batchvarov VN. Measurement, interpretation, and clinical potential of QT dispersion. Journal of the American College of Cardiology. 2000; 36(6):1749-66. doi: 10.1016/s0735-1097(00)00962-1.

3. Vázquez-Seisdedos CR, Neto JE, Marañón Reyes EJ, Klautau A, Limão de Oliveira RC. New approach for T-wave end detection on electrocardiogram: Performance in noisy conditions. Biomed Eng Online. 2011; 77. doi: 10.1186/1475-925X-10-77.

4. Erikssen G, Liestøl K, Gullestad L, Haugaa KH, Bendz B, Amlie JP. The Terminal Part of the QT Interval (T peak to T end): A Predictor of Mortality after Acute Myocardial Infarction. Ann Noninvasive Electrocardiol. 2012; 17(2):85-94. doi: 10.1111/j.1542474X.2012.00493.x. 
5. Helmy H, Abdel-Galeel A, Taha Kishk Y, Mohammed Sleem K. Correlation of corrected QT dispersion with the severity of coronary artery disease detected by SYNTAX score in non-diabetic patients with STEMI. Egypt Hear J. 2017; 69(2):111-117. doi: 10.1016/j. ehj.2016.12.001.

6. Alabd MA, El-Hammady W, Shawky A, Nammas W, El-Tayeb M. QT Interval and QT Dispersion in Patients Undergoing Hemodialysis: Revisiting the Old Theory. Nephron Extra. 2011; 1.

7. $\mathrm{Wu}$ VC, Lin LY, Wu KD. QT interval dispersion in dialysis patients. Nephrology. 2005; 10(2):109-112. doi: 10.1111/j.14401797.2005.00391.x.

8. Okin PM, Devereux RB, Howard B V., Fabsitz RR, Lee ET, Welty TK. Assessment of QT interval and QT dispersion for prediction of all-cause and cardiovascular mortality in American Indians: The strong heart study. Circulation. 2000; 101(1):61-66. doi: 10.1161/01. cir.101.1.61.

9. Rodríguez-Jiménez AE, Cruz-Inerarity H, Negrín-Valdés T, Fardales-Rodríguez R, Chávez-González E. Corrected QT-interval dispersion: An electrocardiographic tool to predict recurrence of myocardial infarction. MEDICC Rev. 2019; 21(2-3):22-25.

10. Ale OK, Ajuluchukwu JNA, Oke DA, Mbakwem AC. QT dispersion in hypertensive Nigerians with and without left ventricular hypertrophy. West Afr J Med. 2013; 32(1):57-61.

11. Demirtaş AO, Urgun OD. Can QT interval prolongation or dispersion detected in a positive exercise ECG test predict critical coronary artery disease? Arch Med Sci - Atheroscler Dis. 2019; 4:e7-e12. doi: 10.5114/amsad.2019.83299.

12. Eslami V, Safi M, Taherkhani M, Adibi A, Movahed MR. Evaluation of QT, QT dispersion, and T-wave peak to end time changes after primary percutaneous coronary intervention in patients presenting with acute ST-elevation myocardial infarction. J Invasive Cardiol. 2013; 25(5):232-234.

13. Dotta G, Fonseca FAH, de Oliveira Izar MC, de Souza MT, Moreira FT, Pinheiro LFM, et al. Regional QT interval dispersion as an early predictor of reperfusion in patients with acute myocardial infarction after fibrinolytic therapy. Arq Bras Cardiol. 2019; 112(1):20-29. doi: 10.5935/abc.20180239.

14. Alici G, Sahin M, Ozkan B, Acar G, Acar RD, Yazicioglu MV, et al. The comparison in reduction of QT dispersion after primary percutaneous coronary intervention according to existence of thrombectomy in ST-segment elevation myocardial infarction. Clin Cardiol. 2013; 36(5):276-9. doi: 10.1002/clc.22109.

15. Alasti M, Adel MH, Torfi E, Noorizadeh M, Bahadoram S, Moghaddam MA, et al. QT dispersion: Does it change after percutaneous coronary intervention? J Tehran Univ Hear Cent. 2011; 6(1):19-23.

16. Matsuno Y, Minatoguchi S, Fujiwara H. Effects of candesartan versus amlodipine on home-measured blood pressure, QT dispersion and left ventricular hypertrophy in high-risk hypertensive patients. Blood Press. 2011; 1:12-9. doi: 10.3109/08037051.2010.532339.

17. Ersoy A, Ersoy C, Guler M, Yildiz A. Losartan and QT Dispersion in Hypertensive Patients on Maintenance Hemodialysis. Med Sci Int Med J. 2014; 3(4):1627-38.

18. Oflaz MB, Balli S, Kibar AE, Ece I, Akdeniz C, Tuzcu V. Effects of carvedilol therapy on cardiac autonomic control, QT dispersion, and ventricular arrhythmias in children with dilated cardiomyopathy. Med Sci Monit. 2013; 19:366-72. doi: 10.12659/MSM.883911.

19. Roden DM. Drug-Induced Prolongation of the QT Interval. N Engl J Med. 2004; 350(10):1013-22. doi: 10.1056/NEJMra032426.

20. Ogiso M, Suzuki A, Shiga T, Nakai K, Shoda M, Hagiwara N. Effect of intravenous amiodarone on QT and T peak-T end dispersions in patients with nonischemic heart failure treated with cardiac resynchronization-defibrillator therapy and electrical storm. J Arrhythmia. 2015; 31(1):1-5. doi: 10.1016/j.joa.2014.01.006.

21. Friedman A, Miles J, Liebelt J, Christia P, Engstrom K, Thachil R, et al. QT Dispersion and Drug-Induced Torsade de Pointes. Cureus. 2021; 13(1):1-10. doi: 10.7759/cureus.12895.

22. Nachimuthu S, Assar MD, Schussler JM. Drug-induced QT interval prolongation: Mechanisms and clinical management. Therapeutic Advances in Drug Safety. 2012. 3(5):241-253. doi: 10.1177/2042098612454283.

23. Prenner SB, Shah SJ, Goldberger JJ, Sauer AJ. Repolarization Heterogeneity: Beyond the QT Interval. Journal of the American Heart Association. 2016; 5:e003607. doi: 10.1161/JAHA.116.003607 